\title{
TYPING DATABASE FOR NOROVIRUSES
}

\author{
E Duizer ${ }^{1}$, Annelies Kroneman ${ }^{1}, \mathrm{~J}$ Siebenga ${ }^{1}$, L Verhoef $^{1}$, H Vennema ${ }^{1}$, M Koopmans ${ }^{1}$, the FBVE network (fbve@rivm.nl) \\ 1. Laboratory for Infectious Diseases, Virology, Department, National Institute for Public Health and the Environment, \\ Bilthoven, the Netherlands
}

\section{The FBVE network}

The FBVE (Food-borne Viruses in Europe) network was initiated during a research project funded by the European Commission (contract QLK1-1999-00594). The aim of the network is to establish a framework for rapid, (pre-publication) exchange of epidemiological, virological and molecular diagnostic data on outbreaks of viral gastroenteritis and acute hepatitis due to hepatitis $A$ and $E$ viruses for both surveillance and research purposes.

Surveillance of (viral) gastroenteritis is not harmonised across Europe as surveillance systems are different in each country. The present FBVE network includes 26 actively participating institutes in 13 countries. Each participating country is represented by at least one virologist and one epidemiologist.

Since the focus of this paper and issue is on typing tools, we do not show extensive documentation on quality of the data (for reference see [1] and [2]).

In September 2001, the FBVE network launched a web-based database for reporting outbreaks of viral gastroenteritis. The FBVE database (www.fbve.nl, password protected) is accessible to all members of the network for online outbreak reporting and to search or download the complete dataset. In addition, sequences can be matched against the complete dataset: the results are presented as a table showing homologous strains detected earlier and their characteristics such as year, country, setting of the outbreak, and mode of transmission. More than 16,000 outbreaks have been entered in the database to date. Another feature is a public genotyping tool that allows 'visitors' to upload partial sequences of specific genomic NoV regions. These sequences are subsequently assigned a NoV genotype (see below).

\section{Norovirus}

Noroviruses (NoV) are small single-strand RNA viruses without an envelope and are classified to the family Caliciviridae. They are highly contagious and are transmitted via faeces and vomit, either directly through contact with infected people or through objects touched by them, or indirectly via contaminated environmental surfaces. The incubation period is about one to three days. The main symptoms of NoV infections are diarrhoea, abdominal pain and vomiting. Infection can occur at all ages, but young children and the elderly are particularly at risk from dehydration. Currently there are no drugs or vaccines that can control or prevent NoV infections [3].
NoV can be divided in five genogroups $(G)$, of which viruses belonging to GI, GII and GIV cause infections in humans. They are further classified into genotypes or genetic clusters, according to the sequence diversity of their capsids. Most prevalent in humans are NoV of GII, and in this group, genotype GII.4 has in recent years been identified as the cause of global epidemics. From time to time, new variants of this genotype appear and rapidly displace the resident dominant variant [4]. There is some evidence for differences in severity of illness [5] and modes and/or efficacy of transmission between viruses that belong to different genotypes. In addition, GII viruses have been found in animals, which raises questions about their zoonotic potential.

\section{Added value of NoV typing}

Genotyping is presently done almost exclusively at the national level as part of surveillance, research projects or outbreak investigations. Several regions of the genome are targeted by a range of RT-PCR assays (Figure). Regions A and C [6]. are most widely used, but other genomic regions have been used as diagnostic targets as well.

For monitoring trends, region $\mathrm{A}$ and $\mathrm{C}$ can be used. However, aggregation of data needs to be done with caution because recombination is quite common in NoV, i.e. a recombinant strain may have region $A$ from one genotype and region $C$ from another. Therefore, recombinant strains could erroneously be labelled as distinct, if region $A$ and region $C$ typing results of different strains are compared and sequences from only one of the regions per strain are available.

\section{Typing tool}

To harmonise the international comparison of NoV sequences, a quick typing tool was set up by the FBVE network. The availability of a common set of reference sequences with universally accepted

F I G U R E

Norovirus genome and sequencing regions $\mathrm{A}$ and $\mathrm{C}$

\begin{tabular}{|l|l|l|l|l|l|}
\hline ORF1 & A & & & \multicolumn{1}{|c|}{} \\
\hline & & & C & ORF2 \\
\hline
\end{tabular}

Open reading frame (ORF) 1: RNA-dependent RNA polymerase ORF 2: Major capsid protein (VP1) 
genotype assignments allows investigators to either rule out or confirm the possibility that a given outbreak is part of a larger (international) outbreak. In the tool, which is publicly accessible at http://www.rivm.nl/bnwww, sequences can be compared to libraries of consensus sequences of regions $A$ and $C$ of different NoV genotypes and variants. The library is regularly updated and newly circulating genotypes or variants are added.

\section{What have we learned through NoV genotyping?}

For outbreak investigations, capsid-based typing, based on ORF2 sequencing, provides the highest discriminatory power and can be used to link cases. This has been done on several occasions, e.g. in a transnational food-borne outbreak of NoV in which contaminated raspberries from Slovenia caused infections in Europe and Canada [1].

However, other examples show that this approach may not be as straightforward, particularly in outbreaks in which the source of contamination of food items is sewage in which case multiple strains, and even different virus families, are often present in the same sample. Under these circumstances, often associated with shellfish-related outbreaks, finding dissimilar sequences in patient samples and in the suspected food does not necessarily provide evidence for the absence of such a link, and more extensive analyses are needed [7].

A comprehensive overview of outbreaks in Europe that were reported with combined epidemiological and virological data showed that particular genotypes seem to be more prevalent in certain settings. For example, the winter peaks in outbreaks in hospitals and homes for the elderly and the global epidemics are mainly caused by Gll.4 strains, whereas in food-borne infections a relatively high number of GI viruses are found. Typing of NoV can indicate the need for follow-up studies and intervention strategies. If GI or non-GII.4 strains are found, food-borne transmission is more likely, and research and intervention strategies can be focused on source tracing and elimination.

\section{Global exchange of norovirus data through noronet}

(noronet@rivm.nl)

A recent expert group meeting, convened by the World Health Organization (WHO), the Food and Agriculture Organization (FAO) and the Organization for Animal Health (OIE), stressed the need for better surveillance of possible food-borne viruses, which presently escape detection. This is a cause for concern because microbiological food safety criteria rely on bacterial indicators that clearly do not reflect virus ecology [8]. Therefore, a global initiative was launched to link researchers from the FBVE network to 19 additional institutes across the world, to discuss harmonisation of methods, and allow comparison of NoV epidemiology across continents.

A retrospective analysis illustrated that the observed GII.4 NoV evolution in Europe reflects a global pattern of emergence of novel variants similar to what has been observed for influenza viruses. This suggests that disease activity in one region could potentially be used as an indicator for "hot seasons" with increased NoV activity in other parts of the world, and data are currently analysed to study if such patterns exist.

\section{Future prospects}

Within the FBVE network, databases have been developed for the surveillance of hepatitis $E$ virus (HEV) and hepatitis A virus (HAV) in Europe to provide a basis to identify possible sources of these viruses. Both databases contain numerous HEV and HAV sequences but do not yet allow for rapid typing. We are presently developing tools to provide customised overviews of the available data via web-based queries.

\section{Acknowledgement}

This work was supported financially by the European Commission, DG Research Quality of Life Program, 6th Framework(EVENT, SP22-CT-2004-502571) and DG SANCO (DIVINE-net, 2003213).

\section{References}

1. Lopman B, Vennema H, Kohli E, Pothier P, Sanchez A, Negredo A, et al. Increase in viral gastroenteritis outbreaks in Europe and epidemic spread of new norovirus variant. Lancet. 2004;363(9410):682-8.

2. Kroneman A, Harris J, Vennema H, Duizer E, van Duynhoven Y, Gray J, et al. Data quality of 5 years of central norovirus outbreak reporting in the European Network for food-borne viruses. J Public Health (Oxf). 2008;30(1):82-90.

3. Green, KY, Kapikian AZ, Chanock RM. Human caliciviruses. In: Knipe DM, Howley PM, Griffin DE, Lamb RA, Martin MA, Roizman B, et al., editors. Fields virology. 4th ed. Philadelphia: Williams \& Wilkins; 2001. p.841-74.

4. Siebenga JJ, Vennema H, Duizer E, Koopmans MP. Gastroenteritis caused by norovirus GGII.4, The Netherlands, 1994-2005. Emerg Infect Dis. 2007;13(1):144-6.

5. van Asten $L$, Siebenga JJ, van den Wijngaard $C$, Verheij $R$, van Vliet $H$, van Pelt $W$, et al. Unexpected increases in illness and death associated with norovirus epidemic peaks. Submitted.

6. Vinjé J, Hamidjaja RA, Sobsey MD. Development and application of a capsid VP1 (region D) based reverse transcription PCR assay for genotyping of genogroup I and II noroviruses, J Virol Methods. 2004;116(2):109-17.

7. Le Guyader FS, Bon F, DeMedici D, Parnaudeau S, Bertone A, Crudeli S, et al. Detection of multiple noroviruses associated with an international gastroenteritis outbreak linked to oyster consumption. J Clin Microbiol. 2006;4(11):3878-82.

8. Lees, D. Viruses and bivalve shellfish. Int J Food Microbiol. 2000 ;59(1-2):81-116.

This article was published on 8 May 2008.

Citation style for this article: Duizer E, Kroneman A, Siebenga J, Verhoef L, Vennema $\mathrm{H}$, Koopmans M, the FBVE network. Typing database for noroviruses. Euro Surveill. 2008;13(19):pii=18867. Available online: http://www.eurosurveillance.org/ViewArticle. aspx?ArticleId $=18867$ 